\title{
Association between Abdominal Fat Distribution and Free Triiodothyronine in a Euthyroid Population
}

\author{
Xiaomin Nie ${ }^{a}$ Yiting $\mathrm{Xu}^{\mathrm{a}} \quad$ Xiaojing $\mathrm{Ma}^{\mathrm{a}} \quad$ Yunfeng Xiao $^{\mathrm{b}} \quad$ Yufei Wang $^{\mathrm{a}}$ \\ Yuqian $\mathrm{Bao}^{\mathrm{a}}$ \\ aDepartment of Endocrinology and Metabolism, Shanghai Jiao Tong University Affiliated \\ Sixth People's Hospital, Shanghai Clinical Center for Diabetes, Shanghai Key Clinical Center \\ for Metabolic Disease, Shanghai Diabetes Institute, Shanghai Key Laboratory of Diabetes \\ Mellitus, Shanghai, China; ${ }^{b}$ Department of Radiology, Shanghai Jiao Tong University \\ Affiliated Sixth People's Hospital, Shanghai, China
}

\section{Keywords}

Visceral fat area - Subcutaneous fat area - Free triiodothyronine - Free triiodothyronine to free thyroxine ratio

\begin{abstract}
Background: Obesity is closely related to thyroid hormones; however, the relationship between abdominal fat distribution and thyroid hormones has rarely been explored. Objectives: This study aimed to explore the relationship between abdominal fat distribution and free triiodothyronine (FT3) and FT3 to free thyroxine (FT4) ratio (FT3/FT4) in a euthyroid population. Methods: The present study enrolled 1,036 participants (age range 27-81 years; 445 men and 591 women). The visceral fat area (VFA) and the subcutaneous fat area (SFA) were determined by magnetic resonance imaging. FT3, FT4, and thyroid-stimulating hormone were measured by an electrochemical luminescence immunoassay. Results: In both men and women, SFA increased according to the increase of FT3 and FT3/FT4 tertiles ( $p$ for trend $<0.05$ ), while VFA did not significantly change. In the multivariate stepwise regression analysis, SFA was independently and positively related to FT3 in both men and women, the standardized $\beta(95 \% \mathrm{Cl})$
\end{abstract}

Xiaomin Nie and Yiting Xu contributed equally to this work.

Xiaojing Ma and Yuqian Bao

Department of Endocrinology and Metabolism

Shanghai Jiao Tong University Affiliated Sixth People's Hospital

600 Yishan Road, Shanghai 200233 (China)

maxiaojing@ @jtu.edu.cn, yqbao@sjtu.edu.cn 
were $0.183(0.094,0.272)(p<0.001)$ and $0.089(0.007,0.171)(p=0.033)$, respectively. Moreover, SFA was independently and positively related to FT3/FT4 in men, the standardized $\beta(95 \% \mathrm{Cl})$ was $0.196(0.101,0.290)(p<0.001)$. However, VFA was not related to either FT3 or FT3/FT4 in both genders. Conclusions: Abdominal subcutaneous fat was independently related to increased FT3 in a euthyroid population.

\section{Introduction}

Over the past few decades, obesity has rapidly spread all over the world. Currently, nearly a third of the world population is overweight or obese, and China ranks first in the size of the obesity population [1]. Obesity, especially central obesity, is closely related to metabolic syndrome, type 2 diabetes, cardiovascular disease, cancer, and other diseases that pose serious health threats to the human body [2].

The pathogenesis of obesity includes an imbalance in energy metabolism. The thyroid hormone is closely related to obesity because of its essential role in modulating energy expenditure and appetite [3]. Free triiodothyronine (FT3), the active form of thyroid hormone, is converted from free thyroxine (FT4) in peripheral tissues. The conversion rate of FT4 to FT3 can be expressed as FT3/FT4. A previous study has found that the serum FT3 level was increased in severely obese individuals [4]. Even in euthyroid populations, the body mass index (BMI), waist circumference (W), and fat percentage (fat $\%$ ) were positively related to FT3 and FT3/FT4 [5-7]. BMI and W are simple parameters to evaluate obesity, and fat\% represents the total body fat content; none of the parameters can describe the status of abdominal fat distribution. Differences in fat distribution pose different risks on the metabolism and the cardiovascular system [8]. Thus, it is important to explore the relationship between fat distribution and FT3 and FT3/FT4.

Magnetic resonance imaging (MRI) and computerized tomography are precise methods to measure abdominal fat and are internationally recognized [9]. Although several studies have explored the relationships between precise fat distribution parameters and FT3 [1013], there exist large discrepancies among the results. In this study, we aimed to explore whether the MRI-measured visceral fat area (VFA) and subcutaneous fat area (SFA) are related to FT3 and FT3/FT4 in a euthyroid population.

\section{Materials and Methods}

\section{Subjects}

The study recruited 1,036 community-based euthyroid participants, with an age range of 27-81 years, in Shanghai, China, from October 2015 to July 2016. Details about participant recruitment and data collection were described in our previous study [14]. All subjects received complete questionnaires, physical examination, laboratory examination, and body content examination. Current smokers were defined as participants who smoked at least one cigarette per day over the past 6 months [15]. The exclusion criteria included a history of diabetes or cardiovascular disease, taking lipid-lowering, hypotensive, or other drugs that might influence the body weight or thyroid function, a history of thyroid disease with a thyroxine supplement or anti-thyroid therapy, severe kidney or liver dysfunction, moderate to severe anemia, malignancy or an intracranial mass lesion, acute infection, taking glucocorticoids, sex hormones, amiodarone or lithium, and abnormal thyroid function.

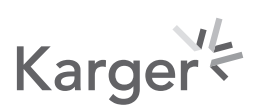


Nie et al.: Subcutaneous Fat and Triiodothyronine

\section{Anthropometric and Biochemical Assessments}

Height, body weight, and blood pressure measurements were performed according to previously described standard methods [14]. BMI was calculated as the body weight (kg) divided by the height squared $\left(\mathrm{m}^{2}\right)$. Systolic blood pressure (SBP) and diastolic blood pressure (DBP) were measured as the mean blood pressure at 3 intervals of 3 min each.

After an overnight fast of $10 \mathrm{~h}$, the subjects received a 75 -g oral glucose tolerance test in the morning. The methods of measuring fasting plasma glucose (FPG), 2-h plasma glucose (2hPG), triglyceride (TG), total cholesterol (TC), high-density lipoprotein cholesterol (HDL-c), low-density lipoprotein cholesterol (LDL-c), glycated hemoglobin A1c (HbA1c), and fasting insulin (FINS) were described in a previous study [14]. Homeostasis model assessment of insulin resistance (HOMA-IR) was used to evaluate the individual level of insulin resistance using the following formula: HOMA-IR = FINS $(\mathrm{mU} / \mathrm{L}) \times$ FPG $(\mathrm{mmol} / \mathrm{L}) / 22.5$ [16].

FT3, FT4, and thyroid-stimulating hormone (TSH) were measured with an electrochemical luminescence immunoassay on a Cobas e601 analyzer (Roche Diagnostics GmbH, Mannheim, Germany). The intra-assay coefficients of variation for FT3, FT4, and TSH were $<7.0 \%,<5.0 \%$, and $<3.0 \%$, respectively. The inter-assay coefficients of variation for FT3, FT4, and TSH were $<8.0 \%,<7.0 \%$, and $<8.0 \%$, respectively. The reference ranges for FT3, FT4, and TSH were 3.10-6.80 pmol/L, 12.00-22.00 pmol/L, and 0.27-4.20 mIU/L, respectively.

\section{Measurement of Abdominal Fat Distribution}

VFA and SFA were determined by a 3.0T MRI scanner (Archiva; Philips Medical System, Amsterdam, The Netherlands); the detailed method was described in a previous study [17].

\section{Statistical Analysis}

All data were analyzed by using the SPSS version 22.0 (SPSS, Inc., Chicago, IL, USA) statistical software. A two-tailed $p$ value $<0.05$ was considered statistically significant. The Kolmogorov-Smirnov test was used to evaluate the normality of variables. Variables with a normal distribution were expressed as the mean \pm standard deviation (SD). Variables with a skewed distribution were expressed as the median (interquartile range). Categorical variables were expressed as frequencies. For normally distributed variables, an independent sample $t$ test was used to compare the difference between two groups, and one-way ANOVA was used for trend analysis. For skewed variables, the Wilcoxon rank-sum test was used to compare the difference between two groups, and the Kruskal-Wallis $\mathrm{H}$ test was used for trend analysis. For categorical variables, $\mathrm{a} \chi^{2}$ test was used for comparison among groups. Spearman correlation analysis was used to explore correlations among variables. Multivariate stepwise regression analysis was used to explore independent factors for FT3 and FT3/FT4. When FT3 or FT3/FT4 was considered as a dependent variable, the original model included SFA, VFA, BMI, and variables that were significantly related to FT3 or FT3/FT4 by the Spearman correlation analysis. GraphPad version 7.0 software (GraphPad Software, Inc., La Jolla, CA, USA) was used for graphing figures.

\section{Results}

\section{Clinical Characteristics of the Study Participants}

The study finally included 1,036 euthyroid community-based participants with a mean age of $59 \pm 8$ years. There were 445 men and 591 women. The median BMI of the subjects was 23.55 $\mathrm{kg} / \mathrm{m}^{2}$ (21.62-25.71); SFA was $164.81 \mathrm{~cm}^{2}$ (128.17-210.53); VFA was $76.79 \mathrm{~cm}^{2}(53.63-104.38)$; FT3 was 4.93 pmol/L (4.60-5.30); FT4 was $16.51 \mathrm{pmol} / \mathrm{L}$ (15.28-17.69); and TSH was 2.16 $\mathrm{mIU} / \mathrm{L}$ (1.55-2.87). Regarding adiposity parameters, the men had a higher BMI and VFA than the 


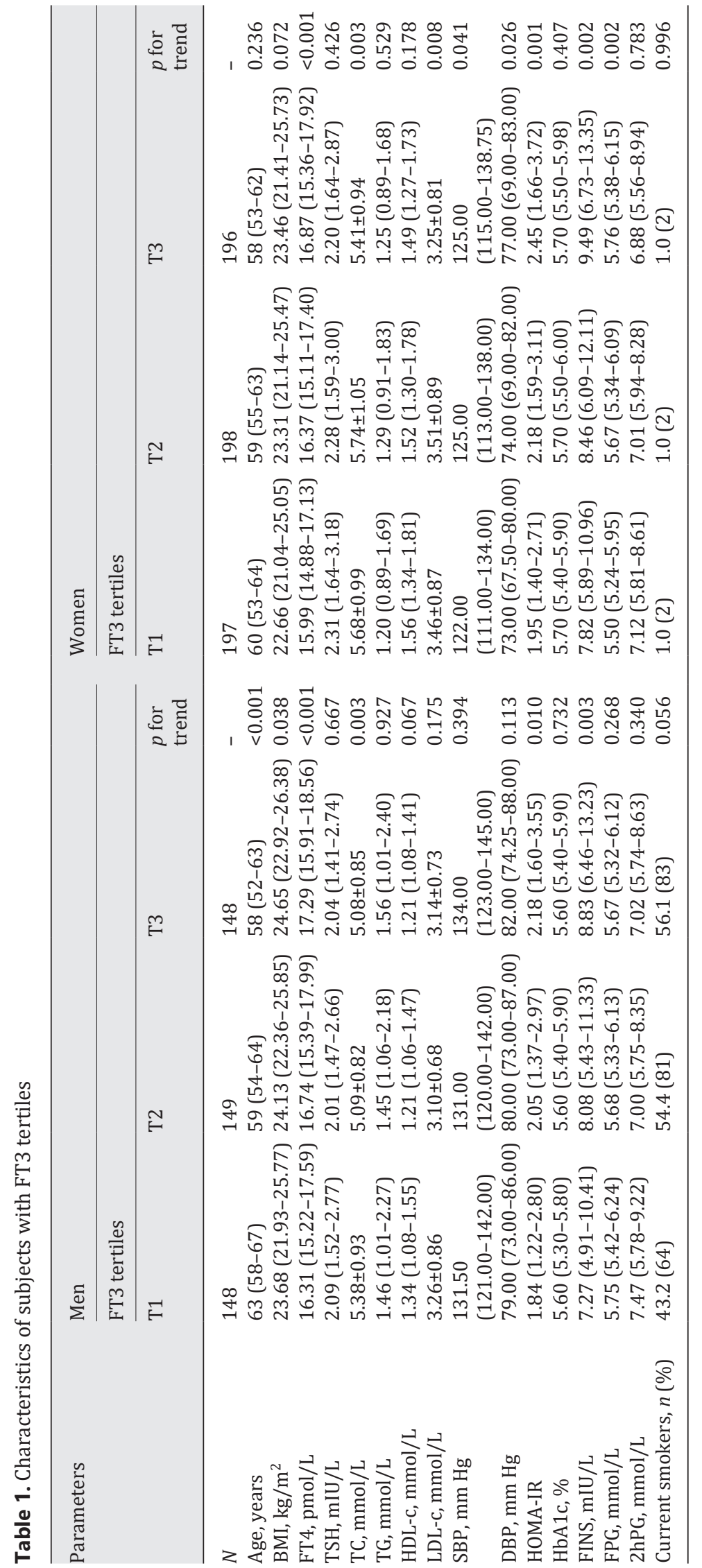

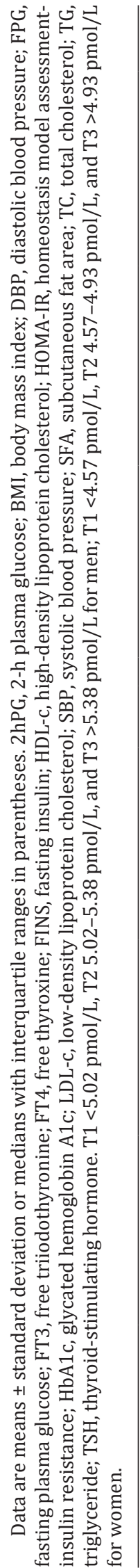




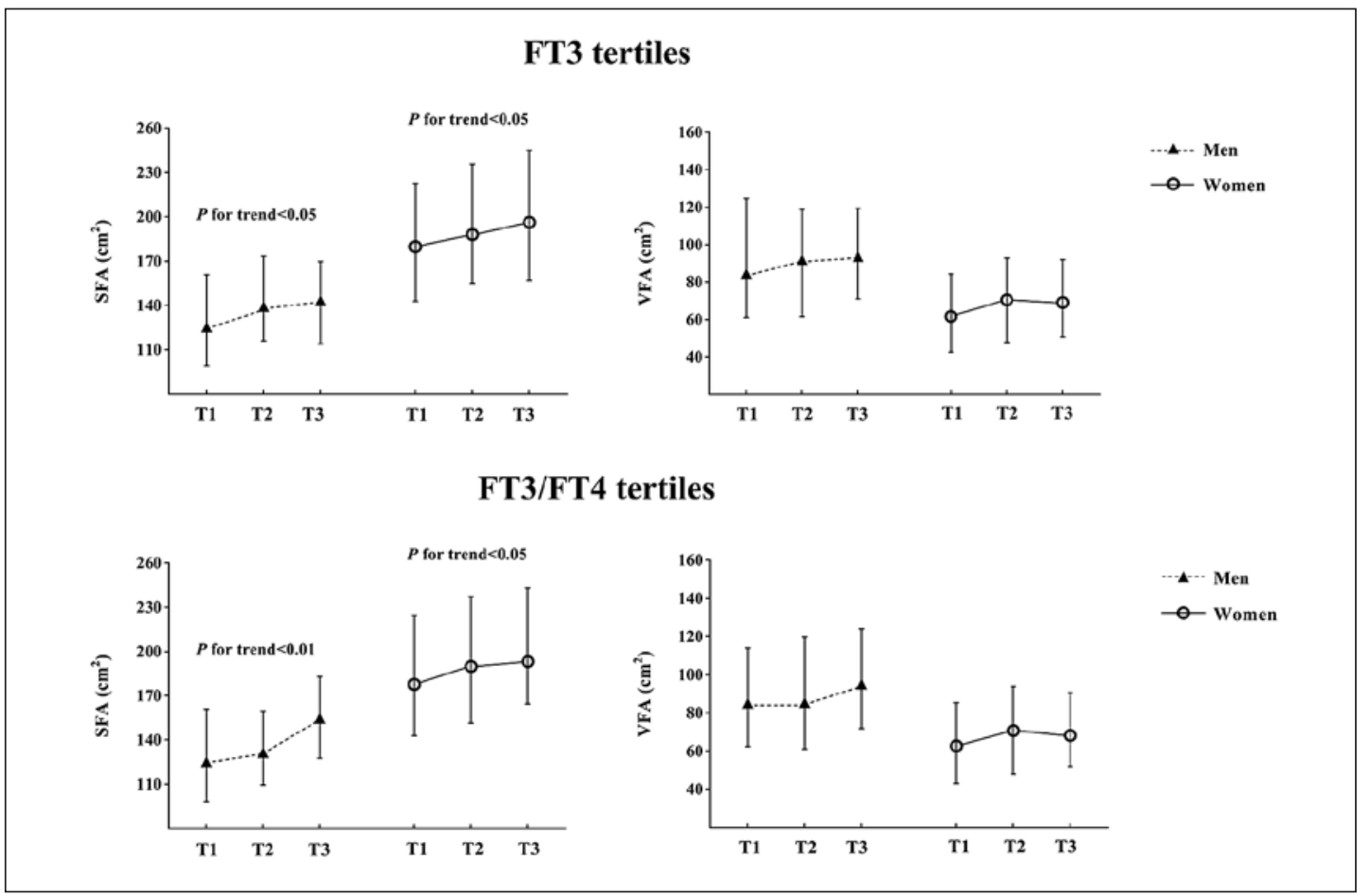

Fig. 1. Relationships of SFA and VFA with FT3 and FT3/FT4 tertiles. Small triangles or circles indicate the median, and the two bars indicate the interquartile range. FT3 tertiles: T1 $<5.02$ pmol/L, T2 5.02-5.38 pmol/L, and T3 > 5.38 pmol/L for men; T1 <4.57 pmol/L, T2 4.57-4.93 pmol/L, and T3 >4.93 pmol/L for women. FT3/FT4 tertiles: T1 <0.29, T2 0.29-0.33, and T3 >0.33 for men; T1 <0.27, T2 0.27-0.31, and T3 > 0.31 for women. The Kruskal-Wallis $\mathrm{H}$ test was used for trend analysis.

women did (24.08 kg/m $\mathrm{m}^{2}$ [22.28-26.07] vs. $23.09 \mathrm{~kg} / \mathrm{m}^{2}$ [21.19-25.36] and $89.00 \mathrm{~cm}^{2}$ [64.46$120.15]$ vs. $67.11 \mathrm{~cm}^{2}$ [46.77-89.07], respectively [both $\left.p<0.001\right]$ ), while the women had a higher SFA than the men did $\left(188.12 \mathrm{~cm}^{2}\right.$ [151.75-234.21] vs. $137.20 \mathrm{~cm}^{2}$ [109.22-169.03] $[p<$ $0.001]$ ). Regarding thyroid hormones, the men had higher FT3 and FT3/FT4 than the women did (5.21 pmol/L [4.89-5.48] vs. $4.74 \mathrm{pmol} / \mathrm{L}$ [4.47-5.07] and $0.31 \mathrm{pmol} / \mathrm{L}$ [0.29-0.34] vs. 0.29 $\mathrm{pmol} / \mathrm{L}$ [0.27-0.32], respectively [both $p<0.001]$ ), while the women had higher TSH than the men did (2.26 mIU/L [1.62-3.02] vs. $2.04 \mathrm{mIU} / \mathrm{L}$ [1.47-2.71] $[p<0.001])$.

Both men and women were divided into 3 groups according to FT3 tertiles (T1 $<5.02$ $\mathrm{pmol} / \mathrm{L}, \mathrm{T} 25.02-5.38 \mathrm{pmol} / \mathrm{L}$, and T3 $>5.38 \mathrm{pmol} / \mathrm{L}$ for men; T1 $<4.57 \mathrm{pmol} / \mathrm{L}, \mathrm{T} 24.57-4.93$ $\mathrm{pmol} / \mathrm{L}$, and T3 $>4.93 \mathrm{pmol} / \mathrm{L}$ for women). In the men, the age and TC decreased with the increase of FT3 tertiles (all $p$ for trend $<0.05$ ), while BMI, FT4, HOMA-IR, and FINS increased with the increase of FT3 tertiles (all $p$ for trend $<0.05$ ). In the women, TC and LDL-c showed downtrends with the increase of FT3 tertiles (all $p$ for trend $<0.05$ ), while FT4, SBP, DBP, HOMA-IR, FINS, and FPG showed uptrends with the increase of FT3 tertiles (all $p$ for trend $<0.05$ ). In the men, TSH, TG, HDL-c, LDL-c, SBP, DBP, HbA1c, FPG, 2hPG, and the percentage of current smokers did not significantly change according to the increase of FT3 tertiles. In the women, the age, BMI, TSH, TG, HDL-c, HbA1c, 2hPG, and the percentage of current smokers did not significantly change with the increase of FT3 tertiles (Table 1).

\section{Distribution of SFA and VFA with FT3 and FT3/FT4 Tertiles}

In both men and women, SFA increased with the increase of FT3 tertiles (all $p$ for trend $<0.05$ ), while VFA did not significantly change. We further divided both men and women into 


\section{Dependent variable: FT3}

Men

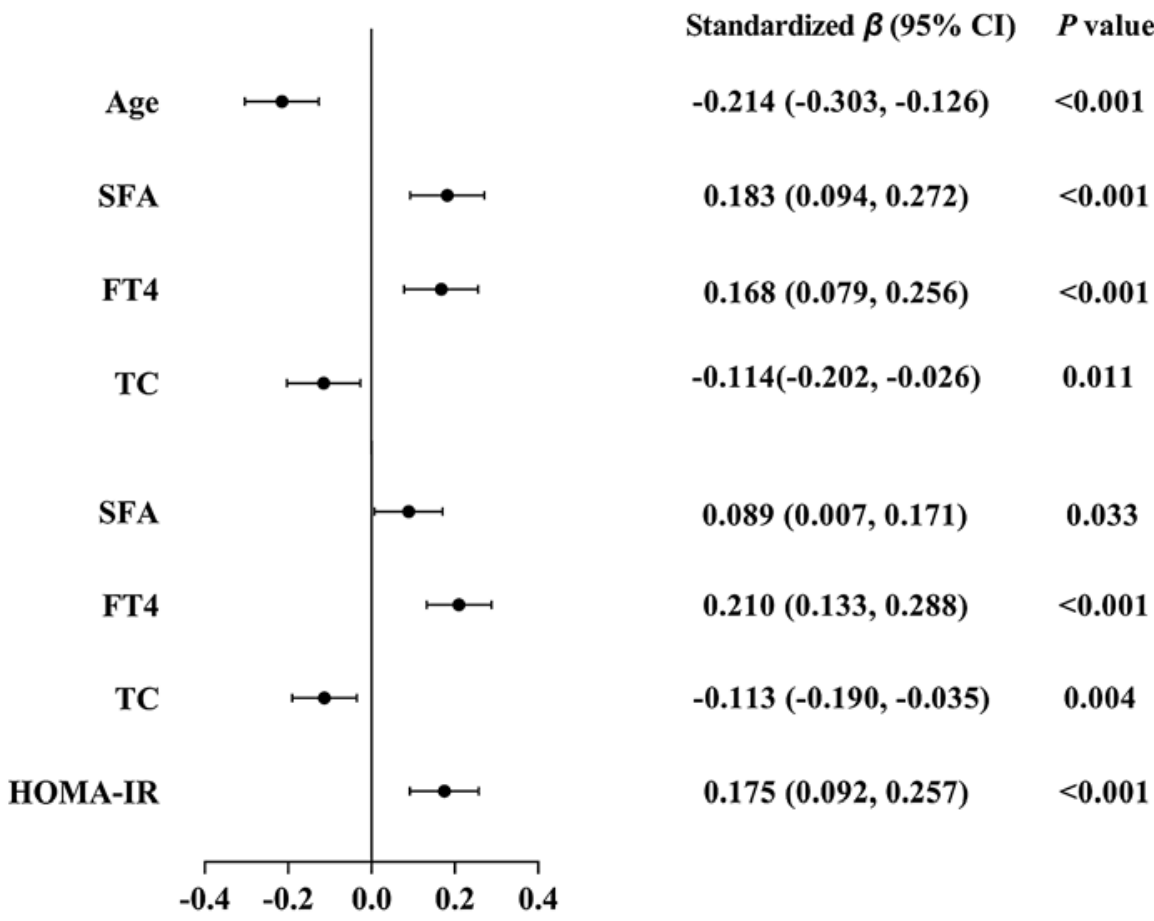

Dependent variable: FT3/FT4

Men

Men

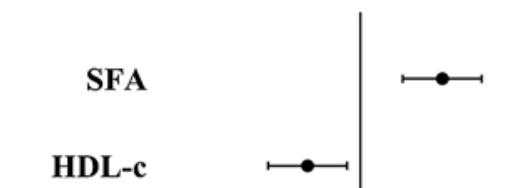

Women

HOMA-IR

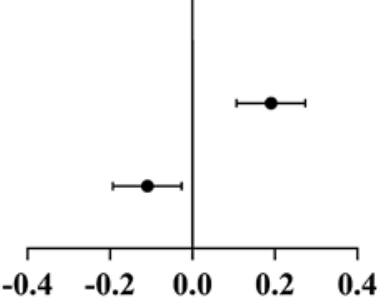

$0.196(0.101,0.290) \quad<0.001$

$-0.126(-0.220,-0.032) \quad 0.009$

$0.191(0.107,0.274) \quad<0.001$

$-0.109(-0.193,-0.026) \quad 0.011$

Fig. 2. Multivariate stepwise regression analyses of FT3 and the FT3/FT4 ratio. In the forest plots, the dots indicate standardized $\beta$, and the two bars indicate 95\% CI. FT3 and FT3/FT4 were ln-transformed. When FT3 or FT3/FT4 was considered a dependent variable, the original model included SFA, VFA, BMI, and variables that were significantly related to FT3 or FT3/FT4 by the Spearman correlation analysis.

3 groups according to FT3/FT4 tertiles (T1 $<0.29$, T2 $0.29-0.33$, and T3 $>0.33$ for men; T1 $<0.27$, T2 $0.27-0.31$, and T3 $>0.31$ for women). In both men and women, SFA increased with the increase of FT3/FT4 tertiles (all $p$ for trend $<0.05$ ), while VFA did not significantly change (Fig. 1). 


\section{Factors Influencing FT3 and FT3/FT4}

We used the Spearman correlation analysis to explore the correlations among variables. In the men, FT3 was positively related to SFA, BMI, FT4, DBP, HOMA-IR, FINS, and the percentage of current smokers, and it was negatively related to age, TC, and HDL-c (all $p<$ 0.05); FT3/FT4 was positively related to VFA, SFA, BMI, HOMA-IR, as well as FINS, and it was negatively related to age and HDL-c (all $p<0.05$ ). In the women, FT3 was positively related to VFA, SFA, BMI, FT4, SBP, DBP, HOMA-IR, FINS, as well as FPG, and it was negatively related to TC and LDL-c (all $p<0.05$ ); FT3/FT4 was positively related to VFA, SFA, BMI, HOMA-IR, FINS, as well as FPG, and it was negatively related to HDL-c (all $p<0.05$ ).

We further used a multivariate stepwise regression analysis to explore the independent factors for FT3 and FT3/FT4. In the men, SFA was independently and positively related to FT3 $(p<0.001)$; in addition, age, TC, and FT4 were independently related to FT3 (all $p<0.05$ ). In the women, SFA was independently and positively related to FT3 ( $p=0.033)$; in addition, TC, HOMA-IR, and FT4 were independently related to FT3 (all $p<0.01$ ).

When FT3/FT4 was the dependent factor, SFA was independently and positively related to FT3/FT4 $(p<0.001)$ in the men. HDL-c was an independent factor for FT3/FT4 in both men and women (all $p<0.05$ ). In the women, HOMA-IR was also an independent factor for FT3/ FT4 $(p<0.001)$ (Fig. 2).

\section{Discussion/Conclusion}

The present study found that the abdominal fat distribution was differentially associated with FT3 and FT3/FT4 in a Chinese euthyroid population. Abdominal subcutaneous fat accumulation was related to increased FT3 in both men and women, and was also related to increased FT3/FT4 in men. However, abdominal visceral fat accumulation was not related to FT3 or FT3/FT4.

Thyroid hormones play essential roles in modulating energy expenditure and appetite [3], and the relationships between thyroid hormones and obesity have always been the focus of attention. Roef et al. [7] found that BMI and W were positively related to FT3 and FT3/FT4 in a healthy euthyroid middle-aged population. According to the National Health and Nutrition Examination Survey of 2007-2008, BMI and W were positively related to FT3 in a euthyroid population [6]. Kim et al. [18] reported that BMI and metabolic syndrome were positively related to total triiodothyronine (TT3) and the TT3 to total thyroxine (TT4) ratio (TT3/TT4). In general, most of the previous studies supported the notion that adiposity parameters were positively related to FT3 and FT3/FT4. However, BMI and W are simple parameters to evaluate obesity, neither could reflect the relationship between abdominal fat distribution and thyroid hormone.

The pathogenesis and progression of obesity are not only related to total fat content, but also to fat distribution. There is a major ontogenetic difference between visceral fat and subcutaneous fat [19]. The body fat distribution can be precisely measured with instruments. Alevizaki et al. [10] measured subcutaneous fat and preperitoneal fat by ultrasound B in 275 nearly healthy subjects, and found that subcutaneous fat was positively related to the TT3 to FT4 ratio, while preperitoneal fat was positively related to TT3. MRI and computerized tomography are precise methods to measure abdominal fat distribution and are recommended by the International Diabetes Federation as gold standards [9]. Nam et al. [11] found that computerized tomography-measured visceral fat was independently and positively related to TT3 in 177 overweight or obese individuals. In the present study, SFA was independently and positively related to FT3 in both men and women and to FT3/FT4 in men; however, VFA was not an independent factor for FT3 or FT3/FT4. The highlight of our study was that precise

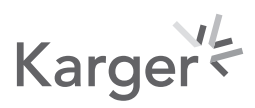


abdominal fat distribution was measured by MRI in a relatively large community-based population. In addition, we excluded individuals with a history of diabetes or cardiovascular disease.

Evidence from basic studies has indicated that subcutaneous fat accumulation may lead to increases in FT3 and FT3/FT4 in several ways. Type 1 iodothyronine deiodinase catalyzes the conversion of FT4 to FT3 in white adipose tissue. A previous study has found that the expression and activity of type 1 iodothyronine deiodinase were both enhanced in white adipose tissue of obese subjects. This effect was more significant in subcutaneous fat tissue than in visceral fat tissue, which suggested that subcutaneous fat tissue might influence the conversion of thyroid hormone in peripheral tissue [20]. The thyroid hormone receptor (THR) is expressed in adipose tissue, and the expression of THR in subcutaneous fat tissue is much higher than that in visceral fat tissue. The expression of THR in adipose tissue was shown to decrease in obesity [21], which suggested that a high level of FT3 in obese individuals might be due to mechanisms similar to those of insulin resistance. Because visceral fat is at a disadvantage in expression of type 1 iodothyronine deiodinase and THR, visceral fat may not have a significant influence on the peripheral metabolism of thyroid hormones.

High levels of FT3 and FT3/FT4 were previously considered adaptive responses to maintain the energy balance in the obese [11]. However, in recent years, some studies have found that a high level of FT3 within the reference range was related to an increased risk of non-alcoholic fatty liver disease and metabolic syndrome [22,23]. Further research is needed to clarify the role of thyroid hormone in metabolic and cardiovascular disease.

Our study has some limitations. Firstly, we did not determine the level of thyroid relatedantibodies and iodine intake. Secondly, owing to the nature of a cross-sectional study, we cannot deduce the causality. Thirdly, the subjects in our study were all Han Chinese adults, and thus the results cannot be generalized to other ethnicities.

In conclusion, abdominal subcutaneous fat was independently related to increased FT3 in a euthyroid population.

\section{Statement of Ethics}

The study was carried out following the rules of the Declaration of Helsinki and approved by the Ethics Committee of the Shanghai Jiao Tong University Affiliated Sixth People's Hospital. All participants provided written informed consent.

\section{Disclosure Statement}

The authors have no conflicts of interest to declare.

\section{Funding Sources}

This work was funded by the National Key R\&D Program of China (2016YFA0502003).

\section{Author Contributions}

The original idea of this study was proposed by Xiaojing Ma and Yuqian Bao. Xiaomin Nie and Yiting $\mathrm{Xu}$ conducted preliminary analyses and wrote the present paper. Xiaomin Nie, Yiting Xu, Yunfeng Xiao, and Yufei Wang performed the experiments. All authors read and approved the final manuscript.

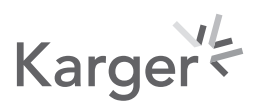


Nie et al.: Subcutaneous Fat and Triiodothyronine

\section{References}

1 Collaboration NC; NCD Risk Factor Collaboration (NCD-RisC). Worldwide trends in body-mass index, underweight, overweight, and obesity from 1975 to 2016: a pooled analysis of 2416 population-based measurement studies in 128.9 million children, adolescents, and adults. Lancet. 2017 Dec;390(10113):2627-42.

2 Bray GA, Heisel WE, Afshin A, Jensen MD, Dietz WH, Long M, et al. The Science of Obesity Management: An Endocrine Society Scientific Statement. Endocr Rev. 2018 Apr;39(2):79-132.

3 Hoermann R, Midgley JE, Larisch R, Dietrich JW. Recent Advances in Thyroid Hormone Regulation: Toward a New Paradigm for Optimal Diagnosis and Treatment. Front Endocrinol (Lausanne). 2017 Dec;8:364.

4 Michalaki MA, Vagenakis AG, Leonardou AS, Argentou MN, Habeos IG, Makri MG, et al. Thyroid function in humans with morbid obesity. Thyroid. 2006 Jan;16(1):73-8.

5 Ren R, Jiang X, Zhang X, Guan Q, Yu C, Li Y, et al. Association between thyroid hormones and body fat in euthyroid subjects. Clin Endocrinol (Oxf). 2014 Apr;80(4):585-90.

6 Kitahara CM, Platz EA, Ladenson PW, Mondul AM, Menke A, Berrington de González A. Body fatness and markers of thyroid function among U.S. men and women. PLoS One. 2012;7(4):e34979.

7 Roef GL, Rietzschel ER, Van Daele CM, Taes YE, De Buyzere ML, Gillebert TC, et al. Triiodothyronine and free thyroxine levels are differentially associated with metabolic profile and adiposity-related cardiovascular risk markers in euthyroid middle-aged subjects. Thyroid. 2014 Feb;24(2):223-31.

8 Abraham TM, Pedley A, Massaro JM, Hoffmann U, Fox CS. Association between visceral and subcutaneous adipose depots and incident cardiovascular disease risk factors. Circulation. 2015 Oct;132(17):1639-47.

9 Alberti KG, Zimmet P, Shaw J. Metabolic syndrome-a new world-wide definition. A Consensus Statement from the International Diabetes Federation. Diabet Med. 2006 May;23(5):469-80.

10 Alevizaki M, Saltiki K, Voidonikola P, Mantzou E, Papamichael C, Stamatelopoulos K. Free thyroxine is an independent predictor of subcutaneous fat in euthyroid individuals. Eur J Endocrinol. 2009 Sep;161(3):459-65.

11 Nam JS, Cho M, Park JS, Ahn CW, Cha BS, Lee EJ, et al. Triiodothyronine level predicts visceral obesity and atherosclerosis in euthyroid, overweight and obese subjects: T3 and visceral obesity. Obes Res Clin Pract. 2010 Oct-Dec;4(4):e247-342.

12 Taneichi H, Sasai T, Ohara M, Honma H, Nagasawa K, Takahashi T, et al. Higher serum free triiodothyronine levels within the normal range are associated with metabolic syndrome components in type 2 diabetic subjects with euthyroidism. Tohoku J Exp Med. 2011 Jul;224(3):173-8.

13 Lambrinoudaki I, Armeni E, Rizos D, Georgiopoulos G, Athanasouli F, Triantafyllou N, et al. Indices of adiposity and thyroid hormones in euthyroid postmenopausal women. Eur J Endocrinol. 2015 Aug;173(2):237-45.

$14 \mathrm{Xu}$ Y, Ma X, Shen Y, Gu C, Tang J, Bao Y. Role of hyperglycaemia in the relationship between serum osteocalcin levels and relative skeletal muscle index. Clin Nutr. 2019 Dec;38(6):2704-11.

15 Yang G, Fan L, Tan J, Qi G, Zhang Y, Samet JM, et al. Smoking in China: findings of the 1996 National Prevalence Survey [J Am Med Assoc]. JAMA. 1999 Oct;282(13):1247-53.

16 Matthews DR, Hosker JP, Rudenski AS, Naylor BA, Treacher DF, Turner RC. Homeostasis model assessment: insulin resistance and beta-cell function from fasting plasma glucose and insulin concentrations in man. Diabetologia. 1985 Jul;28(7):412-9.

$17 \mathrm{Xu}$ Y, Ma X, Pan X, He X, Xiao Y, Bao Y. Correlations between serum concentration of three bone-derived factors and obesity and visceral fat accumulation in a cohort of middle aged men and women. Cardiovasc Diabetol. 2018 Nov;17(1):143.

18 Kim HJ, Bae JC, Park HK, Byun DW, Suh K, Yoo MH, et al. Triiodothyronine Levels Are Independently Associated with Metabolic Syndrome in Euthyroid Middle-Aged Subjects. Endocrinol Metab (Seoul). 2016 Jun;31(2): 311-9.

19 Chau YY, Bandiera R, Serrels A, Martínez-Estrada OM, Qing W, Lee M, et al. Visceral and subcutaneous fat have different origins and evidence supports a mesothelial source. Nat Cell Biol. 2014 Apr;16(4):367-75.

20 Ortega FJ, Jílková ZM, Moreno-Navarrete JM, Pavelka S, Rodriguez-Hermosa JI, Kopeck Ygrave J, et al. Type I iodothyronine $5^{\prime}$-deiodinase mRNA and activity is increased in adipose tissue of obese subjects. Int J Obes. 2012 Feb;36(2):320-4.

21 Nannipieri M, Cecchetti F, Anselmino M, Camastra S, Niccolini P, Lamacchia M, et al. Expression of thyrotropin and thyroid hormone receptors in adipose tissue of patients with morbid obesity and/or type 2 diabetes: effects of weight loss. Int J Obes (Lond). 2009 Sep;33(9):1001-6.

22 Kim HJ, Bae JC, Park HK, Byun DW, Suh K, Yoo MH, et al. Association of triiodothyronine levels with future development of metabolic syndrome in euthyroid middle-aged subjects: a 6-year retrospective longitudinal study. Eur J Endocrinol. 2017 Apr;176(4):443-52.

23 van den Berg EH, van Tienhoven-Wind LJ, Amini M, Schreuder TC, Faber KN, Blokzijl H, et al. Higher free triiodothyronine is associated with non-alcoholic fatty liver disease in euthyroid subjects: the Lifelines Cohort Study. Metabolism. 2017 Feb;67:62-71. 